
\title{
25 Research Soure \\ Differential Effects of Bariatric Surgery on Plasma Levels of ANGPTL3 and ANGPTL4.
}

\section{Simone Bini}

Sapienza University of Rome

\section{Laura D’Erasmo}

Sapienza University of Rome

\section{Brenno Astiarraga}

University of Pisa

Ilenia Minicocci

Sapienza University of Rome

Maria Palumbo

University of Pisa

Valeria Pecce

Sapienza University of Rome

Luca Polito

Sapienza University of Rome

\section{Alessia Costanzo}

Sapienza University of Rome

Rebecca A. Haeusler

Columbia University

\section{Marcello Arca}

Sapienza University of Rome

\section{Ele Ferrannini}

CNR Institute of Clinical Physiology

Stefania Camastra ( $\nabla$ stefania.camastra@unipi.it )

University of Pisa

\section{Research Article}

Keywords: ANGPTL3, ANGPTL4, Bariatric Surgery, Type 2 diabetes mellitus, insulin sensitivity, lipid metabolism.

Posted Date: November 12th, 2021

DOI: https://doi.org/10.21203/rs.3.rs-1017161/v1 
License: (c) (i) This work is licensed under a Creative Commons Attribution 4.0 International License. Read Full License 


\section{Abstract}

Introduction: Angiopoietin-like protein 3 (ANGPTL3) and 4 (ANGPTL4) are regulators of triglycerides storage and utilization. Bariatric surgery (BS) determines profound changes in adipose tissue composition and energy metabolism. We evaluated the impact of BS on plasma levels of ANGPTL3 and ANGPTL4.

Method: Twenty-seven morbidly obese subjects with or without type 2 diabetes (T2D) underwent Roux-en$Y$ gastric bypass (RYGB) and 18 patients with advanced T2D received Biliopancreatic Diversion (BPD). Fasting ANGPTLs levels, insulin sensitivity (evaluated by euglycemic hyperinsulinemic clamp), total bile acids (TBA) and free fatty acids (FFA) were measured at baseline and 1year after surgery.

Results: Both surgical procedures resulted in fat mass loss, improved glucose control, and a 2-fold increase of insulin sensitivity. ANGPTL4 levels decreased significantly with both RYGB $(26.6 \pm 0.6$ to 24.4 $\pm 0.3 \mathrm{ng} / \mathrm{mL}, p=0.001)$ and BPD (27.9 \pm 1.5 to $24.0 \pm 0.5 \mathrm{ng} / \mathrm{mL}, p=0.003)$. In contrast, ANGPTL3 concentrations did not change after RYGB but rose following BPD $(225 \pm 20$ to $300 \pm 15 \mathrm{ng} / \mathrm{mL}$, $p=0.003$ ). By multiple regression analysis, changes in ANGPTL4 were independently associated with those of blood glucose, $p=0.0169$ ) whereas changes in ANGPTL3 after BPD were associated with variations in FFA $(p=0.008)$ and insulin sensitivity $(p=0.0427)$.

Discussion: Circulating ANGPTL4 is reduced by BS probably due to the loss of fat mass and improved insulin sensitivity. Conversely, ANGPTL3 levels increased after BPD, presumably because of the metabolic changes induced by the malabsorptive effect of this surgical procedure.

\section{Introduction}

Angiopoietin-like protein 3 (ANGPTL3) and angiopoietin-like protein 4 (ANGPTL4) are regulators of triglyceride-rich lipoprotein (TRL) metabolism ${ }^{1}$. ANGPTL3 is a secreted glycoprotein expressed almost exclusively by the liver, while ANGPTL4 is produced mainly by the liver and the adipose tissue ${ }^{2}$, both angiopoietins act as negative regulators of the lipoprotein lipase (LPL) ${ }^{3,4}$. More recently, it has been demonstrated that the inhibitory activity of ANGPTL3 and 4 is also modulated by another protein belonging to the angiopoietin-like family: the angiopoietin-like protein 8 (ANGPTL8) ${ }^{5}$. ANGPTL8, secreted by the liver in response to feeding, is able to form complexes with both ANGPTL3 (in a 3:1 molar ratio) and ANGPTL4 (in 1:1 molar ratio); the ANGPTL3/8 complexes are 100-times more potent in LPL inhibition than ANGPTL4/8 complexes, thus establishing a possible hierarchical mechanism in regulating LPL-activity in different tissues ${ }^{6}$.

LPL plays a fundamental role in the catabolism of circulating TRL, such as chylomicrons and very lowdensity lipoproteins (VLDL), by catalyzing the hydrolysis of their triglyceride (TG) content, thus leading to the release of free fatty acids (FFA). Consistently, the overexpression of ANGPTL3 and 4 in mice caused hypertriglyceridemia ${ }^{7}$, and the genetically-determined complete deficiency of ANGPTL3 in humans is 
associated with increased LPL activity, low fasting concentration of plasma TG and increased removal of TRL during the postprandial phase ${ }^{8,9}$.

The function of ANGPTLs goes probably beyond the effect in regulating plasma lipoprotein metabolism as they may be involved in the balance of whole-body energy by influencing lipid substrate trafficking (mainly FFA) and adipose tissue physiology ${ }^{2}$. Available evidence exploring the action of ANGPTLs in adipose tissue is scarce and most published studies are based on murine models. Indeed, contradictory

evidence has been reported on circulating levels of ANGPTL3 and 4 in obese subjects ${ }^{10-12}$. In this scenario, where novel ANGPTL3-lowering therapies will be shortly available as lipid lowering therapy ${ }^{13}$, clinical studies concerning angiopoietin-like proteins variations in extreme metabolic conditions are of uttermost importance, in order to select specific populations where ANGPTL3-lowering would produce the greatest benefit. As an example in case of leptin-deficient lipodystrophy, characterized by the alteration of adipose tissue distribution, plasma levels of ANGPTL3 have been reported to be markedly elevated ${ }^{14}$. At the same time, leptin replacement therapy with Metreleptin produced a significant decrease in plasma ANGPTL3 and TG levels ${ }^{14}$. Of note, some studies have shown that ANGPTL3 can be involved in carbohydrate metabolism ${ }^{15,16}$, but the relation of ANGPTL3 and 4 with parameters of glucose metabolism have not been firmly established.

We have chosen bariatric surgery (BS) as a model of profound metabolic changes in adipose tissue, ${ }^{17}$ as well as in hormonal status ${ }^{18}$ and energy substrate metabolism ${ }^{19}$. The aim of the study was gaining information on the relationship between adipose tissue, energy balance and ANGPTLs regulation. We measured ANGPTL3 and ANGPTL4 levels in obese and diabetic patients before and after BS. By this approach, we intended to verify whether: 1 ) the reduction of adipose mass caused by BS may lead to changes in circulating ANGPTLs and 2) these effects are dependent on the type of BS and may be related to some parameters of glucose and lipid metabolism.

\section{Results}

\section{Baseline characteristics}

The baseline characteristics of the study participants, grouped according to obesity and type 2 diabetes (T2D) status are summarized in Table 1. As compared to obese T2D, non-obese T2D subjects were older and leaner, and showed higher fasting glucose, FFA, and triglyceride concentrations but lower fasting insulin and HDL-cholesterol levels. Insulin sensitivity ( $M$ value) and circulating levels of ANGPTL3 and ANGPTL4 did not differ significantly across groups (Table 1). In the pooled data from all participants, baseline ANGPTL3 levels were positively related to $M$ value $(r=0.37, p=0.02)$, whereas baseline ANGPTL4 levels were inversely related to $M$ value $(r=-0.37, p=0.03)$. 
Table 1

- Baseline characteristics of the study subjects.

\begin{tabular}{|c|c|c|c|}
\hline & Obese NGT & Obese T2D & non-Obese T2D \\
\hline Number (F/M) & $11(10 / 1)$ & $16(11 / 5)$ & $18(7 / 11)$ \\
\hline Age (years) & $43 \pm 2$ & $50 \pm 2^{\S}$ & $56 \pm 1^{*}$ \\
\hline Body weight (kg) & $145 \pm 6$ & $132 \pm 7$ & $78 \pm 2^{\star}$ \\
\hline $\mathrm{BMI}\left(\mathrm{kg} \cdot \mathrm{m}^{-2}\right)$ & $52.7 \pm 1.9$ & $49.8 \pm 2.1$ & $28.2 \pm 0.6^{*}$ \\
\hline Fasting glucose (mmol/L) & $5.2 \pm 0.1$ & $7.2 \pm 0.6^{\S}$ & $12.1 \pm 0.4^{*}$ \\
\hline Fasting insulin (pmol/L) & 138 [112] & 126 [112] & $64[45]^{\star}$ \\
\hline FFA (mEq/L) & $0.60 \pm 0.05$ & $0.70 \pm 0.04$ & $0.84 \pm 0.04^{\star}$ \\
\hline Total cholesterol (mmol/L) & $4.34 \pm 0.18$ & $4.44 \pm 0.30$ & $5.31 \pm 0.39$ \\
\hline Triglycerides (mmol/L & $1.13 \pm 0.15$ & $1.73 \pm 0.46$ & $1.99 \pm 0.16^{*}$ \\
\hline HDL-cholesterol (mmol/L) & $1.23 \pm 0.12$ & $0.98 \pm 0.07$ & $1.17 \pm 0.07 *$ \\
\hline LDL-cholesterol (mmol/L) & $2.61 \pm 0.17$ & $2.70 \pm 0.24$ & $3.25 \pm 0.34$ \\
\hline $\mathrm{TBA}(\mu \mathrm{mol} / \mathrm{L})$ & $1.62[3.06]$ & $2.57[2.76]$ & $1.65[1.62]$ \\
\hline$M$ value $\left(\mu \mathrm{mol} \cdot \mathrm{min}^{-1} \cdot \mathrm{kg}_{\mathrm{FFM}}{ }^{-1}\right)$ & $25.6 \pm 3.2$ & $23.3 \pm 3.4$ & $20.6 \pm 0.9$ \\
\hline ANGPTL3 (ng/ml) & $256 \pm 20$ & $275 \pm 23$ & $225 \pm 17$ \\
\hline ANGPTL4 (ng/ml) & $27.0 \pm 0.8$ & $25.2 \pm 1.0$ & $27.2 \pm 1.0$ \\
\hline
\end{tabular}

\section{After Surgery changes}

Table 2 compares anthropometric and metabolic parameter before and 1 year after BS. Regardless of the BS procedure used, all patients achieved significant BMI, fasting glucose and insulin reduction, together with an improvement in total and LDL cholesterol levels. Only Roux-en-Y gastric bypass (RYGB) patients, however, showed a significant increase in HDL-C and a reduction in serum TG. Both BS techniques were associated with a significant increase in total biliary acids (TBA), which was particularly pronounced in subjects undergoing BPD; patients in the latter group also experienced a decrease in FFA concentrations $(p=0.009)$. As expected, both RYGB and BPD resulted in a marked improvement of insulin sensitivity (Mvalue). 
Table 2

- Anthropometric and laboratory parameters pre-surgery vs 1-year post surgery.

\begin{tabular}{|c|c|c|c|c|c|c|}
\hline & RYGB & & & BPD & & \\
\hline & $\begin{array}{l}\text { Pre- } \\
\text { surgery }\end{array}$ & $\begin{array}{l}\text { Post- } \\
\text { surgery }\end{array}$ & $\mathrm{p}$ & $\begin{array}{l}\text { Pre- } \\
\text { surgery }\end{array}$ & $\begin{array}{l}\text { Post- } \\
\text { surgery }\end{array}$ & $\mathrm{p}$ \\
\hline $\mathrm{N}$ & 27 & 27 & - & 15 & 15 & - \\
\hline Age (years) & $47 \pm 2$ & - & - & $57 \pm 1^{\S}$ & - & - \\
\hline Weight $(\mathrm{kg})$ & $137 \pm 5$ & $93 \pm 4$ & $<0.0001$ & $78 \pm 3^{\S}$ & $64 \pm 2^{*}$ & 0.0007 \\
\hline $\mathrm{BMI}\left(\mathrm{kg} \cdot \mathrm{m}^{-2}\right)$ & $\begin{array}{l}50.9 \pm \\
1.5\end{array}$ & $34.5 \pm 1.1$ & $<0.0001$ & $\begin{array}{l}28.2 \\
\pm 0.7 \S\end{array}$ & $\begin{array}{l}23.2 \pm \\
0.7^{*}\end{array}$ & 0.0007 \\
\hline Fasting glucose (mmol/L) & $6.4 \pm 0.4$ & $5.0 \pm 0.1$ & $<0.0001$ & $\begin{array}{l}12.3 \pm \\
0.5^{\S}\end{array}$ & $7.3 \pm 0.4^{\star}$ & 0.0007 \\
\hline Fasting insulin (pmol/L) & $137[120]$ & $44[22]$ & $<0.0001$ & $61[52] \S$ & $34[9]$ & 0.0007 \\
\hline $\mathrm{FFA}(\mathrm{mEq} / \mathrm{L})$ & $\begin{array}{l}0.68 \\
{[0.22]}\end{array}$ & $0.62[0.18]$ & ns & $\begin{array}{l}0.85 \\
{[0.24]}\end{array}$ & $\begin{array}{l}0.80 \\
{[0.27]}\end{array}$ & 0.009 \\
\hline $\begin{array}{l}\text { Total cholesterol } \\
(\mathrm{mmol} / \mathrm{L})\end{array}$ & $\begin{array}{l}4.40 \pm \\
0.19\end{array}$ & $\begin{array}{l}4.04 \pm \\
0.11\end{array}$ & 0.02 & $\begin{array}{l}5.28 \pm \\
0.42\end{array}$ & $\begin{array}{l}3.52 \pm \\
0.12^{\star}\end{array}$ & 0.009 \\
\hline Triglycerides (mmol/L) & $\begin{array}{l}1.48 \pm \\
0.27\end{array}$ & $\begin{array}{l}0.99 \pm \\
0.07\end{array}$ & 0.002 & $\begin{array}{l}1.99 \pm \\
0.18\end{array}$ & $\begin{array}{l}1.96 \pm \\
0.27^{\star}\end{array}$ & ns \\
\hline HDL-cholesterol (mmol/L) & $\begin{array}{l}1.09 \pm \\
0.07\end{array}$ & $\begin{array}{l}1.27 \pm \\
0.07\end{array}$ & 0.02 & $\begin{array}{l}1.18 \pm \\
0.07\end{array}$ & $\begin{array}{l}1.06 \pm \\
0.07^{\star}\end{array}$ & ns \\
\hline LDL-cholesterol (mmol/L) & $\begin{array}{l}2.66 \pm \\
0.15\end{array}$ & $\begin{array}{l}2.33 \pm \\
0.08\end{array}$ & 0.03 & $\begin{array}{l}3.21 \pm \\
0.36\end{array}$ & $\begin{array}{l}1.59 \pm \\
0.07 *\end{array}$ & 0.006 \\
\hline $\mathrm{TBA}(\mu \mathrm{mol} / \mathrm{L})$ & $\begin{array}{l}2.36 \\
{[3.02]}\end{array}$ & $4.30[6.14]$ & 0.005 & $\begin{array}{l}1.55 \\
{[1.93]}\end{array}$ & $\begin{array}{l}7.11 \\
{[8.03]}\end{array}$ & 0.002 \\
\hline $\begin{array}{l}\text { M value } \\
\left(\mu \mathrm{mol} \cdot \mathrm{min}^{-1} \cdot \mathrm{kg}_{\mathrm{FFM}}{ }^{-1}\right)\end{array}$ & $\begin{array}{l}19.8 \\
{[19.0]}\end{array}$ & 41.9 [10.3] & $<0.0001$ & $19.4[2.7]$ & $33.9[9.1]^{\star}$ & 0.0007 \\
\hline
\end{tabular}

ANGPTL4 levels were significantly decreased in both groups: from $26.6 \pm 0.6$ to $24.4 \pm 0.3 \mathrm{ng} / \mathrm{mL}$ $(p=0.001)$ in the RYGB and from $27.9 \pm 1.5$ to $24.0 \pm 0.5 \mathrm{ng} / \mathrm{mL}(p=0.003]$ in the BPD group (Figure $1 \mathrm{~A})$. In contrast, ANGPTL3 levels did not show any changes in RYGB patients (from $256 \pm 20$ to $228 \pm 28 \mathrm{ng} / \mathrm{mL}$; $p=n s$ ), whereas it was significantly increased (from $225 \pm 20$ to $300 \pm 15 \mathrm{ng} / \mathrm{mL}, p=0.003$ ) in BPD-treated patients) (Figure 1B).

Table 3 shows a multiple regression model for ANGPTL4 variation after bariatric surgery. As both RYGB and BPD patients showed a reduction in ANGPTL4 levels 1-year after surgery, data from both groups was pooled together in this model. After adjusting for age, and for BMI and $\mathrm{M}$ value changes, the main 
determinant of ANGPTL4 changes after surgery was plasma glucose variation. Changes in plasma glucose explained $58 \%$ of ANGPTL4 reduction after $B S\left(R^{2}=0.278 ; p=0.06\right)$ (Table 3$)$. In a multiple regression model including age, and surgery-induced changes in $M$ value, FFA, and TBA, the decrease in FFA and the increase of M were the principal determinants of the ANGPTL3 increase after BPD (Table 4). This result was confirmed in stepwise regression, in which variation of FFA and M-value were the first and second variables forced to enter into the model ( $p=0.0005$; $F$ value 23.2 for FFA, $F$ value 4.2 for M-value variation). In the proposed model, after correction for age and TBA variation, changes in FFA and insulin sensitivity explains respectively $61 \%$ and $35 \%$ of ANGPTL3 increase after BPD $\left(R^{2}=0.781 ; p=0.0024\right)$ (Table 4).

Table 3

- $\triangle$-ANGPTL4 multiple regression analysis

\begin{tabular}{|lllll|}
\hline \multicolumn{5}{|c|}{$\Delta$-ANGPTL4 } \\
& $\beta$ & Std $\beta$ & t-value & $\mathrm{p}$ \\
\hline$\Delta$-Glucose & 0.736 & 0.586 & 2.561 & 0.0169 \\
\hline$\Delta$-M value & 0.115 & 0.366 & 1.755 & 0.0916 \\
\hline$\Delta$-BMl & 0.091 & 0.233 & 0.978 & 0.3375 \\
\hline
\end{tabular}

Table 4

$\triangle$-ANGPTL3 multiple regression analysis

\begin{tabular}{|lllll|}
\hline \multicolumn{5}{|c|}{$\Delta$-ANGPTL3 } \\
\hline & $\beta$ & Std $\beta$ & t-value & $p$ \\
\hline$\Delta$-FFA & -239 & 0.608 & -3.304 & 0.0080 \\
\hline$\Delta$-M value & 4.533 & 0.350 & 2.321 & 0.0427 \\
\hline$\Delta$-TBA & 3.659 & 0.261 & 1.445 & 0.1790 \\
\hline Age & -2.219 & 0.103 & 0.679 & 0.5123 \\
\hline
\end{tabular}

\section{Discussion}

To the best of our knowledge, this is the first study investigating the effect of BS on plasma concentrations of ANGPTL3 and ANGPTL4. We included both NGT and T2D obese patients to gauge the effect of surgery over a broader range of insulin/glucose abnormalities associated with obesity and to evaluate the differential effect of RYGB and BPD on ANGPTLs.

The main findings of this study are: $a$ ) both BPD and RYGB cause a reduction in ANGPTL4 levels; $b$ ) ANGPTL3 increases after BPD but not after RYGB; c) the increase in ANGPTL3 after BPD is significantly associated with the concomitant reduction in FFA. In addition, we found that NGT individuals with morbid 
obesity have similar levels of ANGPTL3 and ANGPTL4 as obese or non-obese T2D patients, and that insulin sensitivity is directly related to ANGPTL3 and inversely related to ANGPTL4.

As expected, BS effectively reduced body weight in all patients, independently of the surgical technique, this paralleled a near doubling of insulin sensitivity. It is noteworthy that TG reduction was obtained with RYGB but not with BPD, which conversely was more effective in reducing TC and LDL-C. As described in a recent meta-analysis, RYGB is the gold standard surgery technique in morbid obesity, but BPD is more effective for the long-term remission of T2D and for cholesterol reduction ${ }^{20,21}$. The latter finding follows from the fact that BPD is a malabsorptive procedure, which causes enhanced bile acid loss; this in turn stimulates bile acid synthesis and an enhanced liver uptake of circulating LDL ${ }^{22}$.

Plasma levels of ANGPTL4 were found to be significantly reduced in all patients treated with either RYGB or BPD: The estimated overall size of this reduction was $\sim 12 \%$. As ANGPTL4 is mainly produced by adipose tissue ${ }^{2,23}$, its reduction can be easily explained by the marked post-surgical reduction of body fat mass. Insulin is a negative regulator of ANGPTL4 synthesis and secretion through the activation of ChREBP and the inhibition of peroxisome-proliferator receptor $a$ and $\gamma$ (PPAR- $a$ and PPAR- $\gamma$ ), which are the main inducers of this angiopoietin ${ }^{2,24}$. The observed reduction in circulating ANGPTL4 may be, therefore, ascribed, at least in part, to the post-surgical improvement of whole-body insulin sensitivity with the attendant fall in glucose and insulin levels (Table 2 and 3). As ANGPTL4 acts as an inhibitor of LPL activity, its reduction following BS might activate TG hydrolysis, thereby leading to TG lowering; this hypothesis was recently proved by Singh et al. ${ }^{25}$ that reported, in a mouse model selectively knocked-out for ANGPTL4, a consistent reduction of circulating lipids, increased hepatic clearance of dietary derived chylomicrons and increased liver and adipose tissue insulin sensitivity ${ }^{25}$. Unfortunately, our study did not include measurements of post-heparin lipase activity (PHLA) before and after surgery so that further studies are needed to clarify this point.

The effect of BS on ANGPTL3 appears to be strictly dependent from surgical procedure employed as its levels were unchanged by RYGB but increased significantly after BPD. Although several studies reported ANGPTL3 to be directly related to BMI (with higher levels in obese people), the positive relation between body fat and ANGPTL3 has not been consistently confirmed ${ }^{11,12}$. We did not find a significant correlation between ANGPTL3 and TG levels, which is in line with some previously reported results ${ }^{26}$ but not with others ${ }^{27}$. This controversial relationship suggests that other factors may affect their mutual regulation. In a previous study, we reported that the relation between plasma levels of ANGPTL3 and TG is not linear 26. In fact, TG levels (and other lipids such as total cholesterol, HDL- and LDL-cholesterol) were strongly correlated with ANGPTL3 only when ANGPTL3 levels are below $60 \mathrm{ng} / \mathrm{dL}$; at higher levels there no longer was a significant association with serum lipids ${ }^{26}$. The synthesis and secretion of ANGPTL3 are finely regulated by multiple factors, primarily by liver $X$ receptor (LXR) ${ }^{4,28}$. While oxysterols and fatty acids are natural ligands of LXR, the receptor also is activated under conditions of intracellular lipid deprivation and abundant intracellular glucose, thereby acting as a glucose sensor, stimulating lipogenesis and inhibiting gluconeogenesis 29,30 . 
Insulin has been shown to be an inhibitor of ANGPTL3 expression. Haridas et al. reported a reduction in plasma ANGPTL3 during a euglycemic hyperinsulinemic clamp in healthy subjects, and in culture medium of $\mathrm{IHH}$ cells treated with insulin ${ }^{16}$. Yilmaz et al. found a direct relationship between HOMA-IR (an indirect index of hepatic insulin resistance) and plasma ANGPTL3 in patients with fatty-liver disease ${ }^{15}$. In our patients, the BPD-induced rise in ANGPTL3 was directly related to changes in insulin sensitivity and inversely related to changes in serum FFA. This might be explained by differences in the intracellular downstream signaling of LXR activation ${ }^{31,32}$. Thus, the post-surgical doubling of insulin sensitivity may raise intracellular glucose concentrations, and activate LXR thereby stimulating lipogenesis and liver lipid export ${ }^{30}$. In BPD patients, the concomitant reduction in cholesterol and bile acids is a double hit for LXR hyperactivation, thereby promoting ANGPTL3 secretion. Consistently, Cinkajzlová et al. found increased levels of serum ANGPTL3 in patients suffering from short-bowel syndrome (SBS) in comparison with healthy controls ${ }^{12}$. Notably, BPD patients can be considered as analog to SBS patients in terms of nutrients absorption and loss.

FFA were found to be negative drivers of ANGPTL3 in the BPD group. Although medium-chain FFA are LXR agonists ${ }^{33}$, only C-10 and C-12 FFA are able to activate LXR as do oxysterols or glucose ${ }^{33}$. Serum FFA decrease is associated with increased adipose tissue insulin sensitivity, resembling a post-prandial state where insulin inhibits adipose tissue lipolysis ${ }^{34,35}$.

In interpreting these results, several limitations must be considered. The number of patients included in the study is small and the assignment of patients to RYGB or BPD was not randomized (due to partially different indications and clinical experience with the two procedures). More importantly, the difference of BMI among RYGB and BPD patients is wide. Finally, plasma levels of ANGPTL8, another angiopoietin-like protein involved in the activation of ANGPTL3, were not investigated.

In summary, BS showed the expected effect in improving the metabolic status of obese patients with or without T2D. While circulating ANGPTL4 was uniformly decreased by surgery, ANGPTL3 remained stable in RYGB patients but increased in BPD patients. This suggests that the chronic nutrient loss and malabsorption associated with BPD may be counterbalanced by the increased production of ANGPTL3 in the liver. It is possible that the decrease in ANGPTL4 (expressed mostly in WAT) might promote the uptake and storage of TG-derived lipids into WAT, whereas the increase in ANGPTL3 (expressed mostly in liver) might potentially slow down lipid uptake into liver.

In this regard, it is worth mentioning that our results highlighted an important relation between FFA, bile acids metabolism and insulin sensitivity with ANGPTL3, thus opening a new view on the complex regulation of ANGPTL3 metabolism, further in vitro and in vivo work is necessary to detail the molecular mechanism underlying ANGPTL3 regulation in extreme metabolic conditions.

\section{Materials And Methods}

\section{Study patients}


The study group included 45 patients eligible for BS due to morbid obesity and/or T2D. The baseline clinical characteristics of these patients have been partially described in a previous report ${ }^{22}$.

In brief, 27 patients with morbid obesity (BMI range 38-62 kg/m2) underwent RYGB; of them, 11 had normal glucose tolerance (NGT) and 16 had T2D (Obese T2D group). Eighteen, non-morbidly obese patients (BMI range 23-33 kg/m2) with decompensated T2D (non-Obese T2D) underwent BPD. Assignment to RYGB or BPD was based on the decision of the treating physician. In non-T2D patients, glucose status was determined by an oral glucose tolerance test (OGTT) according to standard procedures ${ }^{36,37}$. In the BPD group, T2D patients were treated with oral glucose-lowering agents and/or insulin (metformin in 6 patients, metformin plus sulfonylurea in 3 patients, and metformin plus insulin in 6 patients). In the RYGB group, 6 T2D patients were treated with diet alone and 10 were on oral glucoselowering agents and/or insulin (metformin in 6 patients and metformin plus sulfonylurea in 3 patients and metformin plus insulin in 1 patient).

Study subjects were studied before surgery (baseline) by measuring biochemical and hormonal parameters as well as by performing a euglycemic-hyperinsulinemic clamp, as described below. All patients in the RYGB group and 15 patients in the BPD group were restudied 1-year after surgery with the same protocol.

The study protocol was approved by the Ethic Committee of the University of Pisa. The nature and purpose of the study were carefully explained to all participants. All subjects were asked to voluntarily participate into the present study and written informed consent was obtained from all participants, research and clinical practices were carried out in accordance with the principles of the Helsinki Declaration of 1964 and its revision of 2018.

\section{Euglycemic-hyperinsulinemic clamp}

All investigations were performed in the morning after an overnight fast. Patients with T2D were asked to stop metformin 1 week and sulfonylureas $48-72 \mathrm{~h}$ before the study; in those on insulin, injections were discontinued $16 \mathrm{~h}$ before the metabolic study (patients on bedtime glargine had been switched to NPH 2 days before the study). The euglycemic-hyperinsulinaemic clamp was performed as already described ${ }^{36}$. In brief, a catheter was inserted into an antecubital vein for infusion of all test substances and another catheter was inserted retrogradely into a vein on the dorsum of the hand for blood drawing. The hand was heated to obtain arterialization of venous blood. At time $-20,-10$, and $0 \mathrm{~min}$, blood samples were obtained from the arterialized vein for the measurement of glucose and insulin. At time 0 , a primedcontinuous insulin (Humulin R; Eli Lilly and Company, Indianapolis, USA) infusion (at a rate of $240 \mathrm{pmol}$ . $\mathrm{min}^{-1} \cdot \mathrm{m}^{-2}$ ) was started and continued for $180 \mathrm{~min}$; plasma glucose levels were measured every $5 \mathrm{~min}$ throughout the clamp. Plasma insulin concentrations were measured every 20 min between 120 and 180 min after the start of insulin infusion. Fat-free mass (FFM) was estimated by electrical bioimpedance (TBF 300; Tanita, Tokyo, Japan). 
Insulin-stimulated glucose disposal ( $\mathrm{M}$ value) was calculated as the mean exogenous glucose infusion rate during the last $40 \mathrm{~min}$ of the clamp corrected for changes in glucose concentration within a distribution volume of $200 \mathrm{~mL}$ per kilogram of body weight. M was expressed per kilogram of FFM (in umol. $\mathrm{min}^{-1} \cdot \mathrm{kg}_{\mathrm{FFM}}{ }^{-1}$ ) and used to estimate the insulin sensitivity ${ }^{18}$.

\section{Analytical methods}

Blood samples were separated by centrifugation at $3000 \times \mathrm{g}$ for $15 \mathrm{~min}$ and stored at $-80^{\circ} \mathrm{C}$ until assays. Plasma glucose was measured by the glucose oxidase technique on a Beckman Glucose Analyzer (Beckman, Fullerton, CA). Plasma insulin was assayed by a specific radioimmunoassay (Linco Research, St. Charles, MO, and MYRIA, Technogenetics, Milan, Italy, respectively). Plasma FFA concentrations were measured spectrophotometrically by an automated procedure (Synchro CX4; Beckman, Brea, CA, USA). Plasma triglycerides and serum high-density lipoprotein (HDL) cholesterol were assayed in duplicate by standard spectrophotometric methods on a Synchron Clinical System CX4 (Beckman Instruments, Fullerton, USA). Total bile acids (TBA) were measured by liquid chromatography-tandem mass spectrometry (LC-MS/MS) (Waters Quattro Micro with Waters 2795 Alliance HPLC). Serum ANGPTL3 and ANGPTL4 levels were measured by sandwich enzyme immunoassays using commercial ELISA kits (RND systems \#DANL30, and Biovendor \#RD191073200R). The assay was performed according to the manufacturer's protocol; sensitivity values were $0.035 \mathrm{ng} / \mathrm{mL}$ for ANGPTL3 and $0.173 \mathrm{ng} / \mathrm{ml}$ for ANGPTL4.

\section{Statistical analysis}

Data are reported as mean \pm SE or median [interquartile range] for variables with non-normal distribution. Group differences were tested by Mann-Whitney $U$ test and Wilcoxon signed rank test. For selected variables, two-way ANOVA for repeated measures was used to compare surgical operations across time of study. Simple associations were tested by calculating Spearman's rank correlation coefficient $(\rho)$, data fitting was performed by non-linear regression techniques. Multivariate analysis was carried out using general linear models; results are given as the standardized regression coefficient. Statistical analyses were performed using Stat View 5.0 software, a $p$ value $\leq 0.05$ was considered significant.

\section{Declarations}

Funding: This research has been partially supported by funds from Sapienza University of Rome (\#000106-19-Arca); the National Institutes of Health (\#R01HL12564 and \#R01HL125649); and a grant from Italian Ministry of Health (RF-2011-02348446).

Disclosures: LD has received personal fees for public speaking, consultancy or grant support from Amryt Pharmaceuticals, Akcea Therapeutics, Pfizer, Amgen and Sanofi; MA has received research grant support from Amryt Pharmaceutical, Amgen, IONIS, Akcea Therapeutics, Pfizer and Sanofi; has served as a consultant for Amgen, Aegerion, Akcea Therapeutics, Regeneron, Sanofi and Alfasigma and received lecturing fees from Amgen, Amryth Pharmaceutical, Pfizer, Sanofi and AlfaSigma. EF reports receiving 
consultancy/speaker fees, outside the present work, from Boehringer Ingelheim, Lilly\&Co., AstraZeneca, and Sanofi.

Other authors have declared no conflict of interest.

Acknowledgements: SB contributed to this paper as part of his Ph.D. studies in Biotechnologies and Clinical Medicine at "Sapienza" University of Rome.

Authors contribution: All authors edited the paper. SB and LD wrote the manuscript; BA and MP performed the in vivo study; IM, VP, LP, AD performed the laboratory analysis; RAH is responsible of the bile acids measures in a blinded fashion and contributed to the discussion. MA is responsible of the ANGPTLs measures, contributed to design the study and discussion and revised the manuscript; EF contributed to design the study and revised the manuscript; SC designed the study, analyzed the data, contributed to write the manuscript and discussion section in particular. All authors approved the final version of this manuscript.

\section{References}

1. Zhang R. The ANGPTL3-4-8 model, a molecular mechanism for triglyceride trafficking. Open Biol. 2016;6(4). doi:10.1098/rsob.150272

2. Bini S, D’Erasmo L, Di Costanzo A, Minicocci I, Pecce V, Arca M. The interplay between angiopoietinlike proteins and adipose tissue: Another piece of the relationship between adiposopathy and cardiometabolic diseases? Int J Mol Sci. 2021;22(2):1-16. doi:10.3390/ijms22020742

3. Sukonina V, Lookene A, Olivecrona T, Olivecrona G. Angiopoietin-like protein 4 converts lipoprotein lipase to inactive monomers and modulates lipase activity in adipose tissue. Proc Natl Acad Sci U S A. 2006;103(46):17450-17455. doi:10.1073/pnas.0604026103

4. Inaba T, Matsuda M, Shimamura M, et al. Angiopoietin-like protein 3 mediates hypertriglyceridemia induced by the liver X receptor. J Biol Chem. 2003;278(24):21344-21351. doi:10.1074/jbc.M213202200

5. Haller JF, Mintah IJ, Shihanian LM, et al. ANGPTL8 requires ANGPTL3 to inhibit lipoprotein lipase and plasma triglyceride clearance. J Lipid Res. 2017;58(6):1166-1173. doi:10.1194/jlr.M075689

6. Chen YQ, Pottanat TG, Siegel RW, et al. Angiopoietin-like protein 8 differentially regulates ANGPTL3 and ANGPTL4 during postprandial partitioning of fatty acids. J Lipid Res. 2020; (317):jlr.RA120000781. doi:10.1194/jlr.ra120000781

7. Köster A, Chao YB, Mosior M, et al. Transgenic angiopoietin-like (Angptl)4 overexpression and targeted disruption of Angpt/4 and Angptl3: Regulation of triglyceride metabolism. Endocrinology. 2005;146(11):4943-4950. doi:10.1210/en.2005-0476

8. Minicocci I, Tikka A, Poggiogalle E, et al. Effects of angiopoietin-like protein 3 deficiency on postprandial lipid and lipoprotein metabolism. J Lipid Res. 2016;57(6):1097-1107.

doi:10.1194/jlr.P066183

Page 12/15 
9. Di Costanzo A, Di Leo E, Noto D, et al. Clinical and biochemical characteristics of individuals with low cholesterol syndromes: A comparison between familial hypobetalipoproteinemia and familial combined hypolipidemia. J Clin Lipidol. 2017;11(5):1234-1242. doi:10.1016/j.jacl.2017.06.013

10. Barja-Fernández S, Folgueira C, Castelao $C$, et al. ANGPTL-4 is associated with obesity and lipid profile in children and adolescents. Nutrients. 2019;11(6). doi:10.3390/nu11061340

11. Garcés MF, Buell-Acosta JD, Rodríguez-Navarro HA, et al. Serum angiopoietin-like 3 levels are elevated in obese non diabetic men but are unaffected during an oral glucose tolerance test. Sci Rep. 2020;10(1). doi:10.1038/s41598-020-77961-8

12. Cinkajzlová A, Mráz M, Lacinová Z, et al. Angiopoietin-like protein 3 and 4 in obesity, type 2 diabetes mellitus, and malnutrition: The effect of weight reduction and realimentation. Nutr Diabetes. 2018;8(1). doi:10.1038/s41387-018-0032-2

13. D’Erasmo L, Bini S, Arca M. Rare Treatments for Rare Dyslipidemias: New Perspectives in the Treatment of Homozygous Familial Hypercholesterolemia (HoFH) and Familial Chylomicronemia Syndrome (FCS). Curr Atheroscler Rep. 2021;23(11):65. doi:10.1007/s11883-021-00967-8

14. Muniyappa R, Abel BS, Asthana A, et al. Metreleptin therapy lowers plasma angiopoietin-like protein 3 in patients with generalized lipodystrophy. J Clin Lipidol. 2017;11(2):543-550. doi:10.1016/j.jacl.2017.02.002

15. Yilmaz Y, Ulukaya E, Atug O, Dolar E. Serum concentrations of human angiopoietin-like protein 3 in patients with nonalcoholic fatty liver disease: Association with insulin resistance. Eur J Gastroenterol Hepatol. 2009;21(11):1247-1251. doi:10.1097/MEG.0b013e32832b77ae

16. Haridas PAN, Soronen J, Sädevirta S, et al. Regulation of angiopoietin-like proteins (ANGPTLs) 3 and 8 by insulin. J Clin Endocrinol Metab. 2015;100(10):E1299-E1307. doi:10.1210/jc.2015-1254

17. Camastra $S$, Vitali $A$, Anselmino $M$, et al. Muscle and adipose tissue morphology, insulin sensitivity and beta-cell function in diabetic and nondiabetic obese patients: effects of bariatric surgery. Sci Rep. 2017;7(1):9007. doi:10.1038/s41598-017-08444-6

18. Camastra S, Manco M, Frascerra S, laconelli A, Mingrone G, Ferrannini E. Daylong pituitary hormones in morbid obesity: effects of bariatric surgery. Int J Obes. 2009;33(1):166-172. doi:10.1038/ijo.2008.226

19. Camastra S, Palumbo M, Santini F. Nutrients handling after bariatric surgery, the role of gastrointestinal adaptation. Eat Weight Disord - Stud Anorexia, Bulim Obes. April 2021. doi:10.1007/s40519-021-01194-5

20. Ding L, Fan Y, Li H, et al. Comparative effectiveness of bariatric surgeries in patients with obesity and type 2 diabetes mellitus: A network meta-analysis of randomized controlled trials. Obes Rev. 2020;21(8). doi:10.1111/obr.13030

21. Xu G, Song M. Recent advances in the mechanisms underlying the beneficial effects of bariatric and metabolic surgery. Surg Obes Relat Dis. 2021;17(1):231-238. doi:10.1016/j.soard.2020.08.028

22. Ferrannini E, Camastra S, Astiarraga B, et al. Increased Bile Acid Synthesis and Deconjugation After Biliopancreatic Diversion. Diabetes. 2015;64. doi:10.2337/db15-0214 
23. Dijk W, Heine M, Vergnes L, et al. ANGPTL4 mediates shuttling of lipid fuel to brown adipose tissue during sustained cold exposure. Elife. 2015;4(OCTOBER2015). doi:10.7554/eLife.08428

24. Kersten S, Mandard S, Tan NS, et al. Characterization of the fasting-induced adipose factor FIAF, a novel peroxisome proliferator-activated receptor target gene. J Biol Chem. 2000;275(37):2848828493. doi:10.1074/jbc.M004029200

25. Singh AK, Chaube B, Zhang X, et al. Hepatocyte-specific suppression of ANGPTL4 improves obesityassociated diabetes and mitigates atherosclerosis in mice. J Clin Invest. 2021;131(17). doi:10.1172/JCl140989

26. Fazio S, Minnier J, Shapiro MD, et al. Threshold effects of circulating angiopoietin-like 3 levels on plasma lipoproteins. J Clin Endocrinol Metab. 2017;102(9):3340-3348. doi:10.1210/jc.2016-4043

27. Li J, Yang Y, Jiao X, et al. The Clinical Role of Angiopoietin-Like Protein 3 in Evaluating Coronary Artery Disease in Patients with Obstructive Sleep Apnea. Cardiovasc Drugs Ther. 2020;34(6):773-780. doi:10.1007/s10557-020-06991-1

28. Reeskamp LF, Tromp TR, Huijgen R, Stroes ESG, Hovingh GK, Grefhorst A. Statin therapy reduces plasma angiopoietin-like 3 (ANGPTL3) concentrations in hypercholesterolemic patients via reduced liver $X$ receptor (LXR) activation. Atherosclerosis. 2020;315:68-75. doi:10.1016/j.atherosclerosis.2020.11.013

29. Mitro N, Mak PA, Vargas L, et al. The nuclear receptor LXR is a glucose sensor. Nature. 2007;445(7124):219-223. doi:10.1038/nature05449

30. Wang B, Tontonoz P. Liver $\mathrm{X}$ receptors in lipid signalling and membrane homeostasis. Nat Rev Endocrinol. 2018;14(8):452-463. doi:10.1038/s41574-018-0037-x

31. Cariello M, Piccinin E, Moschetta A. Transcriptional regulation of metabolic pathways via lipidsensing nuclear receptors. Cell Mol Gastroenterol Hepatol. February 2021.

doi:10.1016/j.jcmgh.2021.01.012

32. lizuka K, Takao K, Kato T, Horikawa Y, Takeda J. ChREBP Reciprocally Regulates Liver and Plasma Triacylglycerol Levels in Different Manners. Nutrients. 2018;10(11):1699. doi:10.3390/nu10111699

33. Bedi S, Hines GV, Lozada-Fernandez V V., et al. Fatty acid binding profile of the liver X receptor a. $J$ Lipid Res. 2017;58(2):393-402. doi:10.1194/jlr.M072447

34. Carpentier AC. 100th anniversary of the discovery of insulin perspective: insulin and adipose tissue fatty acid metabolism. https://doi.org/101152/ajpendo006202020. 2021;320(4):E653-E670. doi:10.1152/AJPEND0.00620.2020

35. Montastier É, Ye RZ, Noll C, et al. Increased postprandial nonesterified fatty acid efflux from adipose tissue in prediabetes is offset by enhanced dietary fatty acid adipose trapping. Am J Physiol Metab. 2021;320(6):E1093-E1106. doi:10.1152/ajpendo.00619.2020

36. Camastra S, Gastaldelli A, Mari A, et al. Early and longer term effects of gastric bypass surgery on tissue-specific insulin sensitivity and beta cell function in morbidly obese patients with and without type 2 diabetes. Diabetologia. 2011;54(8):2093-2102. doi:10.1007/s00125-011-2193-6 
37. Association AD. Diagnosis and classification of diabetes mellitus. Diabetes Care. 2010;33(SUPPL. 1):S62-S69. doi:10.2337/dc10-S062

\section{Figures}

A

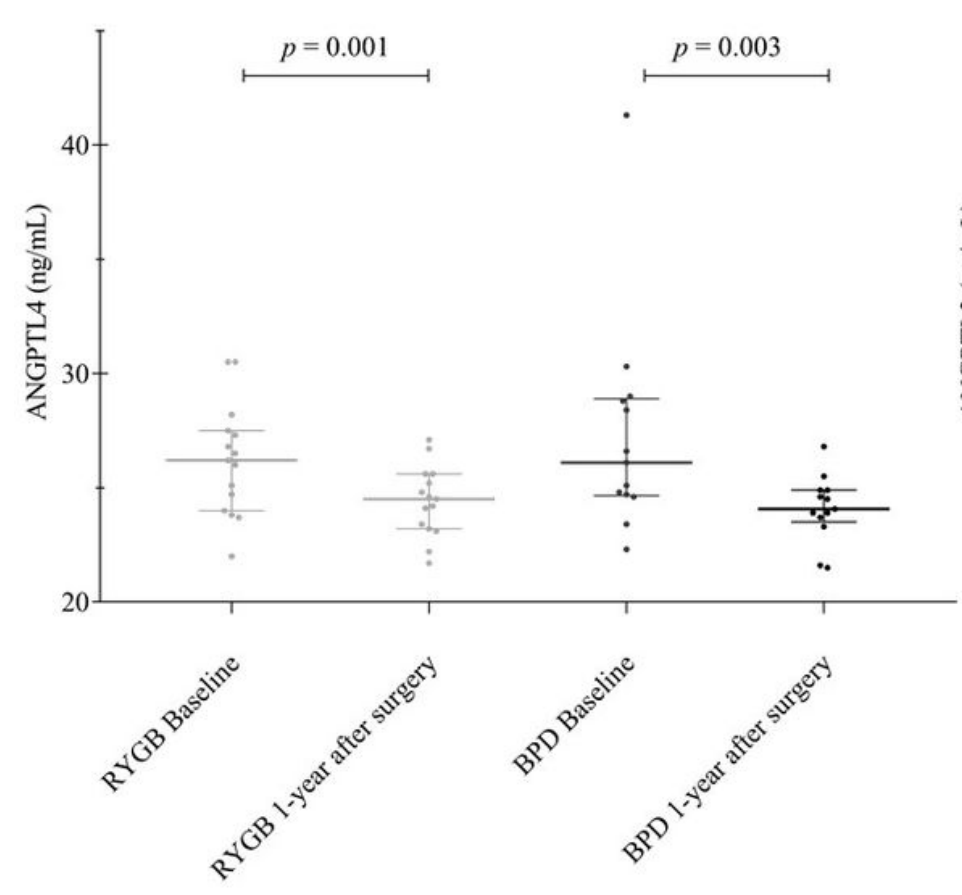

B

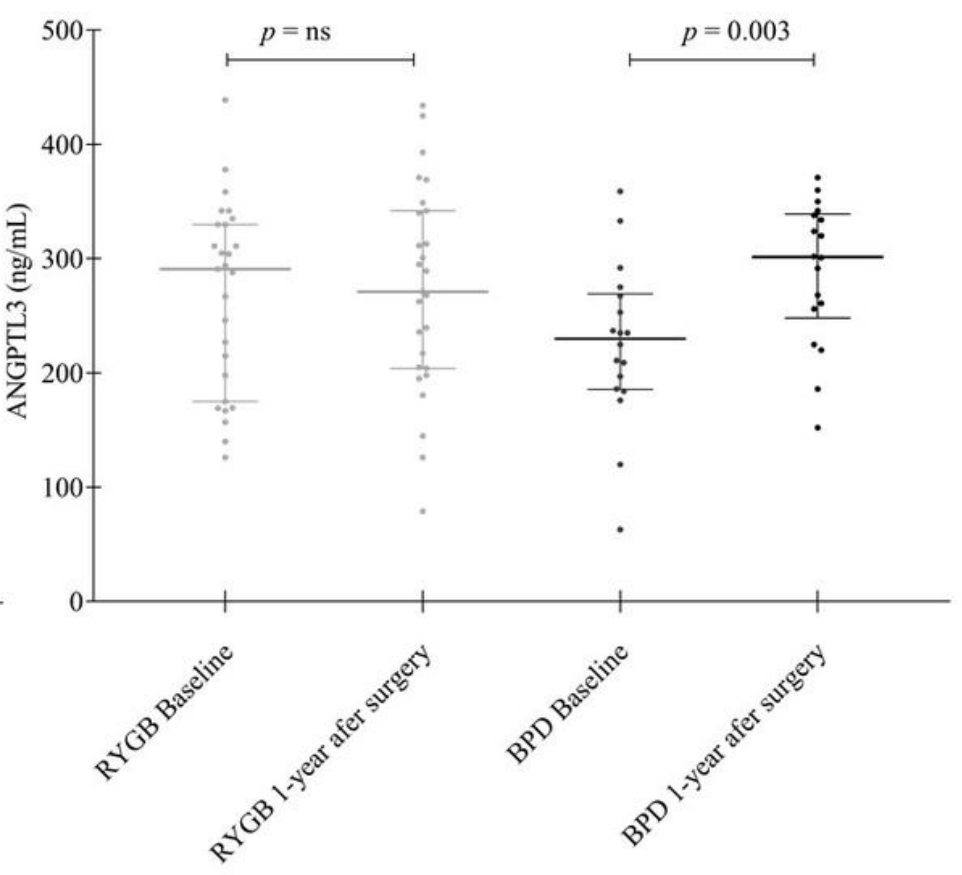

Figure 1

ANGPTL4 and ANGPTL3 variation in RYGB and BPD surgeries. Box plot of ANGPTL4 (A) and ANGPTL3 (B) levels in Roux-en-Y Gastric Bypass (RYGB) surgery and Biliopancreatic Diversion (BPD). 